\title{
PERSPECTIVA Y FORMACIÓN DE OBJETOS EN EL DISCURSO. A PROPÓSITO DE DOS VECES JUNIO, DE MARTÍN KOHAN
}

\author{
María Isabel FILINICH \\ Universidad de Puebla
}

\section{$\mathbf{I}$}

\section{ntroducción}

Hablar implica siempre, entre otros actos, poner en perspectiva aquello que constituye el objeto considerar la toma de posición - perceptiva, cognitiva, axiológica - que implica el acto de asumir la palabra para comunicar algo al destinatario del discurso.

Podría pensarse que el estudio de la toma de posición involucra fundamentalmente al sujeto y que para abordar esta problemática es necesario partir de su configuración. Sin embargo, ya ha sido señalado acertadamente que para comprender desde qué ángulo se focaliza lo observado es necesario reconocer cómo está constituido el objeto del discurso.

Es éste precisamente el sentido de una de las premisas del estudio del punto de vista emprendido por A. Rabatel: «Así, mientras el acercamiento tradicional de la focalización consistía en investigar el "focalizador", "quién ve" o "quién sabe", nuestro acercamiento consiste en investigar las huellas lingüísticas de un punto de vista en el modo de donación del referente de... el objeto percibido» (1998: 58 , la traducción es nuestra $)^{1}$. Por su parte, J. Fontanille señalará que «es preciso preguntarse si el punto de vista no se encuentra en primer lugar en el objeto, antes de estar en el sujeto» (1994: 40), para luego hacer hincapié en la interacción y la constitución recíproca entre sujeto y objeto en las operaciones discursivas del «efecto punto de vista»: «en suma, el punto de vista se definiría como un estado de cosas que es el producto de una interacción dificultosa, comprometida, incluso conflictiva, entre el sujeto y el objeto de la percepción. Precisamente es la imperfección de esta interacción la que permite "significar" al punto de vista» (43).

Con fundamento en esta concepción básica de la puesta en perspectiva como interacción entre sujeto y objeto, aquí nos interesará abordar, entre los diversos aspectos del modo como el discurso

\footnotetext{
1 «Ainsi, alors que l'approche traditionnelle de la focalisation consistait à rechercher le "foyer", "qui voit" ou "qui sait", notre approche consiste à rechercher les traces linguistiques d'un PDV [point de vue] dans le mode de donation du référent de... l'objet perçu» (A. Rabatel, 1998: 58).
} 
configura el proceso de percepción, el de la formación de objetos, en el entendido de que a partir del reconocimiento de la forma del objeto es posible reconstruir los otros aspectos del proceso de percepción que subyace en todo discurso. Además, desde el momento en que la percepción puede ser analizada como un fenómeno interactivo y, por ende, como un campo en el que se generan las primeras formas de la significación, el modelo de la enunciación se presenta como un sustento idóneo para dar cuenta también de la actividad perceptiva que implica todo ejercicio discursivo.

Puede afirmarse entonces que, al poner en escena la percepción, se asiste a un doble proceso enunciativo conformado, por una parte, por el despliegue de la actividad perceptiva, en la que interviene un observador, el sujeto de la percepción, así como aquello que es objeto de la observación (posiciones a las que habría que agregar las operaciones propias de la percepción, que serán abordadas en este trabajo); y por otra parte, al estar la percepción puesta en discurso, esa escena es enmarcada por otra en la que interviene un enunciador, un enunciatario y aquello que es destinado a este último, el objeto del discurso, entre lo que cabe siempre incluir la escena perceptiva subyacente (además, claro está, de las operaciones propias de la enunciación: desembrague, embrague, aspectos éstos que no trataremos aquí).

\section{La escena perceptiva: el papel del objeto}

Un modelo enunciativo de la percepción muestra, en primer lugar, que los actantes que intervienen poseen ambos propiedades de agentes: sujeto y objeto desempeñan un papel activo. Fontanille (1987: 74) ha enfatizado este rasgo proponiendo para estas instancias las categorías de observador e informador respectivamente: así, el observador es aquel que «sabe que hay algo que saber», mientras que el informador es el que «sabe que hay algo que hacer saber». Con todo, hay que tener en cuenta que esta subjetivación de ambos actantes no es simétrica: mientras que la del observador es inmediata y resulta de la puesta en marcha del proceso de significación, la del informador es mediatizada por el saber del observador, pues es éste el que le atribuye un carácter de sujeto, siendo así «una construcción subjetiva de segundo grado» (Fontanille, 1988: 15).

Es necesario pensar, entonces, a todo objeto de observación como aquello a lo que un observador le puede atribuir la capacidad de «hacer saber», lo cual implica, claro está, el «hacer no saber». En este sentido es ilustrativo el caso del camuflaje, estudiado recientemente en una perspectiva semiótica, entre otros, por Paolo Fabbri (2013), quien llama la atención sobre el complejo juego de estrategias que involucra: la desaparición (tornarse invisible) o la transformación en otro (un animal que adopta la forma de un vegetal, por ejemplo) pueden ser comprendidas como formas de manipulación de la mirada por parte del informador. Desde el mimetismo animal hasta el uso del color en transportes y uniformes militares, pasando por el arte y la moda, en todos los casos lo que cuenta es «la credibilidad del simulacro ofrecido por el otro, los juegos interactivos y los regímenes de creencia y de sospecha que se desencadenan» (2013: 768). De aquí que, tratándose de la puesta en perspectiva, el punto de partida para reconstruir la escena perceptiva sea precisamente el informador, aquello que, en otros 
términos, es objeto de la percepción, pues será su potencial para convocar posibles recorridos interpretativos y su contraste con aquellos seleccionados por el observador los que pongan de manifiesto la significación realizada.

Detengámonos en un fragmento de la novela Dos veces junio, de Martín Kohan, para examinar la potencialidad de lo que es puesto bajo la mira y los recorridos de observación seleccionados:

(1) El cuaderno de notas estaba abierto, en medio de la mesa. Había una sola frase escrita en esas dos páginas que quedaban a la vista. Decía: “¿A partir de qué edad se puede empesar a torturar a un niño?” [...]

Descubrí que al lado del cuaderno de notas, estaba la birome con la que esa nota había sido escrita. Una birome rota en el extremo, evidentemente porque alguien descargaba sus nervios mordiendo el plástico ingrato. Tomé esa birome, tratando de no tocar la parte rota: tal vez estuviera húmeda todavía. Mi pulso por entonces ya era bueno. Era capaz de enhebrar un hilo hasta en las agujas más pequeñas. Por eso pude agregar el trazo faltante a la letra ese, y que no se notara que había habido una corrección posterior. Desde siempre parecía haber sido una zeta, tal la gracia de la colita que yo adosé en la parte de abajo de la letra. Ahora la ese era una zeta, como corresponde.

Pocas cosas me contrarían tanto como las faltas de ortografía (Kohan, 2002: 11-12).

Así da inicio la novela. Aquello que es puesto bajo la mira es una frase hallada en un cuaderno de notas (enseguida sabremos que ese cuaderno está junto a un teléfono en una dependencia militar). La frase, además de ser el hilo conductor de acciones centrales del relato, es objeto de observación, en este fragmento, por parte del actor que asume también la función de narrar la historia. Colocada al inicio, con anterioridad al proceso de percepción que desencadena en el personaje, la frase muestra potencialmente, en su desnudez y crudeza, un amplio espectro de significaciones que daría lugar a diversos recorridos interpretativos. Diríamos que la frase, en tanto informador, hace saber incluso más de lo que dice. Sin embargo aquí, el observador (que es también actor de los acontecimientos) elige ver la frase en su aspecto gramatical, más específicamente, ortográfico: la frase tiene un error, una falla en su grafía y eso es lo que ha movido al personaje a observarla y repararla. De los aspectos posibles de ser observados, uno ha sido aislado y éste ha concentrado toda la atención del observador. Colmar la observación con ese detalle es también, por parte del observador, impedirse o, al menos, dispensarse de ver el resto. De aquí que digamos que aquello que se observa habla de lo observado pero fundamentalmente del propio observador. La frase es objeto de un proceso de aspectualización, de una suerte de descomposición en partes o más bien, aspectos, uno de los cuales se magnifica para volverse el todo. Así, una parte reemplaza al todo y lo que se ve puede constituirse, como en este caso, en un obstáculo que enmascara el resto.

La misma frase será objeto de otra mirada por parte de otro actor del relato; anotada por el cabo Leiva, esa anotación es evaluada por el sargento Torres:

(2) El sargento Torres pegó una trompada sobre la mesa y por poco no se cerró el cuaderno donde se leía. Era conveniente, y nadie lo ignoraba, no hacer enojar al sargento Torres. Ahora rugía sus palabras. «¡Cabo Leiva!», bramó, «ésta no es manera de registrar una comunicación!». [...]

El sargento silabeó: «A-par-tir-de-qué-dad-se-pue-dem-pe-zar-a-tor-tu-rar-aun-ni-ño».

Después aplastó el cuaderno con un manotazo.

«¿Qué es esto?», exclamó. «¿Una adivinanza?»

«No, mi sargento», decía el cabo.

« ¿Una prueba de ingenio?»

«No, mi sargento.» 
« Una pregunta filosófica?»

«No, mi sargento.»

«¿ $\mathrm{O}$ acaso está preparando el examen de ingreso para la Facultad de Medicina?»

«No, mi sargento.»

Recién entonces el sargento se aplacó. Le dijo al cabo Leiva que en lo sucesivo nunca dejara de registrar las comunicaciones en la forma debida: aclarando quién tomaba la comunicación, quién la dirigía y a quién la dirigía; y en el caso de que el mensaje tuviera cierta urgencia, como parecía suceder con este mensaje, era su obligación destacarlo con el simple trámite de escribir debajo la palabra «urgente», preferentemente en letra de imprenta, y en lo posible subrayándola dos o tres veces, hasta cuatro de ser necesario (35-36).

Otra falla es puesta en evidencia: el mensaje no está bien registrado, está incompleto, falta información. Para este nuevo observador, el escándalo de la nota reside en que muestra la incompetencia de quien la tomó, en que no se han consignado los datos correctamente. Vemos así que otro aspecto de la frase es ahora puesto de relieve: su registro sobre el papel. El pasaje de la recepción oral del mensaje a su registro escrito lo ha despojado de información relevante para el lector de la nota (distinto del destinatario de la frase).

La frase volverá a ser objeto de observación por parte de su mismo destinatario, el doctor Mesiano, quien enfrenta al doctor Padilla, destinador de la frase, en los siguientes términos:

(3) «Su ignorancia, doctor Padilla. ¿A quién se le ocurre que lo que cuenta en esto es la edad?

¿Qué pensaba? ¿En la maduración afectiva? ¿En el desarrollo psicomotriz? Aquí lo que cuenta es la masa corporal, doctor. Vea lo imprecisa que era su pregunta.»

«No discutamos esto delante del conscripto, doctor, aunque usted le tenga confianza.» 124).

«Es el peso lo que importa, y no la edad. Hasta un estudiante de medicina lo hubiese sabido» (123-

Lo que ahora irrita de la frase a este nuevo observador es la ineptitud de quien la formula: para la «ciencia médica» que el doctor Mesiano pretende representar en esa circunstancia, el criterio de la edad es irrelevante, la pregunta carece de sentido en el dominio médico y debe ser reformulada para que pueda tener respuesta.

Todas estas escenas perceptivas puestas en discurso son, a su vez, objeto de percepción del destinatario del relato (del narratario), cuyo ángulo de percepción es más amplio y puede observar tanto lo observado como a los observadores. Dejamos en suspenso estas cuestiones para poder detenernos ahora en las operaciones a que es sometido todo objeto de percepción y que se ponen claramente de manifiesto en los fragmentos citados.

\section{La construcción del objeto: noción y operaciones}

Aquello que es puesto en discurso como objeto de percepción se conforma mediante una serie de operaciones que intentaremos precisar.

Pero antes de eso, es necesario hacer algunas consideraciones acerca de la noción misma de objeto. Su sentido deberá desprenderse de su significado de 'cosa': en la actitud espontánea, recuerda Borel (1991), se confunden cosa y objeto; pero en una perspectiva teórica, la cosa es irreductible al 
discurso, es aquello no pensado que hay en el pensamiento, mientras que el objeto es siempre una forma, no una cosa, pues el discurso traza, esboza, in-forma, recorriendo aquello que hace presente.

Como toda forma, el objeto no posee una permanencia inalterable. En esto, el objeto del cual aquí tratamos, comparte rasgos con el objeto de carácter teórico. El sugerente título del texto editado por L. Daston (2014), Biografías de los objetos científicos, conduce a pensar en la trayectoria vital de los objetos de las ciencias, esto es, en su emergencia, prominencia, productividad, desaparición... de los objetos, tanto de las ciencias naturales como de las humanidades, puras y aplicadas. La biografía no sería así un género exclusivo de los seres humanos sino que también puede pensarse en la biografía de los objetos, en la generación, desarrollo, formas cambiantes, y extinción de aquello que es puesto bajo la mira. Idea ésta que sigue la estela del pensamiento de Foucault (1970), quien habla de «la formación de los objetos» como de un proceso que es fruto de un conjunto de relaciones entre instancias de emergencia, de delimitación y de especificación.

Pero la forma que tiene el discurso cotidiano de hacer presente los objetos se diferencia de la organización lógica propia del objeto teórico para acercarse a la idea de clase-objeto derivada de la mereología: en este sentido, un objeto puede entenderse como una clase «en la cual pueden entrar un número a priori indeterminado y no calculable de elementos, que poseen como único punto en común el tener todos algo que ver con la denominación genérica de la clase. Nombraré a esos elementos los aspectos del objeto» (Apothéloz, 1983: b5, la traducción es nuestra) ${ }^{2}$. La noción es suficientemente amplia como para que puedan entrar en ella los más diversos elementos asociados a un objeto: sus partes, propiedades, y también los recuerdos que evoca, deseos, y connotaciones en general. Además, para que sea considerado un objeto de percepción es necesario que se efectúe sobre él un recorrido, lo que implica tanto el pasaje de uno a otro aspecto como también detenerse y fijar la mirada y el pensamiento sobre algún aspecto en particular.

Volviendo al fragmento (1) diremos que de la frase en cuestión se toma uno solo de sus aspectos posibles, la materialidad gráfica, para hacer de ese aspecto el centro de la observación: pareciera que aquí hay un procedimiento inverso al que caracteriza a la descripción pues si esta última se define como la expansión de un pantónimo (Hamon, 1991), en el caso que nos ocupa se realiza una operación por descarte de rasgos, siendo sólo uno de ellos el que toma el lugar del todo. Un aspecto vale por el todo pero no al modo del aspecto representativo de la totalidad sino a la manera como un detalle puede encubrir y ocupar el lugar del todo. Este enmascaramiento del todo por el privilegio exclusivo de una parte pone de manifiesto que los aspectos del objeto generalmente son sometidos no sólo a las posibilidades perceptuales de un campo de presencia determinado sino también a un sistema de valores que actúa ya desde la misma percepción, seleccionando y jerarquizando lo percibido.

\footnotetext{
$2 \ll[\ldots]$ dans laquelle peuvent entrer un nombre a priori indéterminé et non calculable d'éléments, qui ont pour seul point commun dávoir tous quelque chose affaire avec la dénomination générique de la classe. Je nommerai ces éléments les aspects de l'objet» (Apothéloz, 1983: b5).
} 
El objeto, lejos de estar dado de antemano, es construido por el discurso y su conformación es inestable, pues queda sometido a constantes reajustes. En esta actividad de construcción del objeto Apothéloz reconoce cuatro operaciones (aunque esta enumeración no es exhaustiva):

i) la aspectualización, por la cual se introducen los diferentes aspectos del objeto - en el sentido amplio arriba explicado- ya sea que se trate de partes, propiedades, funciones, o bien, elementos, otros objetos o carga semántica adherida al objeto;

ii) la tematización, que transforma un aspecto en una nueva cla se-objeto al volverlo tema de un nuevo despliegue; procedimiento que puede entenderse como un efecto de zoom producido por un acercamiento de la visión y por lo tanto como una magnificación de una parte cuyos aspectos pueden exigir otra trayectoria de la mirada;

iii) la afectación, que transforma, primero, un objeto en aspecto, para luego atribuirlo a un nuevo objeto; aquello que desde un punto de vista constituye un objeto, al variar el foco de observación puede ser visto como un aspecto de otro objeto; se trata de un procedimiento que aparece en la resolución de enigmas, en las explicaciones o en la inversión entre pantónimo y despliegue en la descripción cuando primero aparece un despliegue de elementos y se cierra con la mención del objeto;

iv) la asimilación, por la cual se relacionan haces de aspectos de dos objetos en principio extraños uno al otro, como en la metáfora o en la comparación; a estos dos modos de la asimilación, Adam y Petitjean (1989) añaden la reformulación como una de las formas que puede asumir la operación de asimilación; la reformulación, el acto de retomar lo dicho con otros términos, ha sido considerada justamente como el resultado de un cambio de perspectiva (Roulet, 1987) marcado en el discurso, aunque no siempre, por conectores tales como «en efecto», «en suma», o bien, en los casos de reformulaciones parafrásticas, mediante «es decir», «en otros términos», etcétera.

Tomando en consideración las posibles operaciones en la formación de objetos de discurso, volvamos ahora sobre el primer fragmento citado. En (1) aquello que es puesto bajo observación, la frase, aparece compuesta, o bien, aspectualizada en una serie de elementos que la vuelven de interés para el observador: está apuntada en un cuaderno de notas, está a la vista, tiene un error ortográfico, ha sido evidentemente apuntada por otro distinto del observador, conserva a su lado la pluma con la que se escribió. Todos estos aspectos hacen a la frase en cuestión, de otro modo difícilmente hubiera provocado la contrariedad de quien la encuentra, aunque, claro está, para que este efecto se produzca también es necesario que quien la encuentra posea una disponibilidad a ser afectado por la incorrección ortográfica (en esto reside, precisamente, el carácter interactivo de la constitución del sujeto y del objeto de percepción: un rasgo de lo observado atrae a una instancia disponible a ser afectada por ese aspecto). 
Este procedimiento de formación de la frase como objeto puesto en perspectiva ilustra la operación de aspectualización (en el sentido de Apothéloz). Ahora bien, dado que el objeto no puede aparecer sino puesto en perspectiva esto hace también que otros aspectos susceptibles de actualizarse queden virtualizados en esta primera aparición del objeto. Esto se pone de manifiesto en los otros dos fragmentos citados puesto que serán otros los aspectos del objeto que se actualizarán: en (2) se ironiza hiperbólicamente a partir del vacío semántico producido por el registro deficiente de la anotación y aparecen así los tipos de textos en los que podría insertarse la frase en cuestión (adivinanza, pregunta filosófica, examen..., forma de la asimilación que hacer ver la frase como uno u otro tipo de texto), para luego hacer emerger otros aspectos ausentes en la nota pero que se juzgan imprescindibles (quién la apunta, quién la dirige, a quién, grado de urgencia); y en (3) otros serán los aspectos realzados, ahora aquellos referidos a la pertinencia disciplinaria de la pregunta que hace aflorar la ignorancia de quien la formula.

Por otro lado, ya lo hemos señalado, esta aspectualización de la frase es seguida por otra operación: uno de los aspectos actualizados concentra el foco de atención del observador, en detrimento de los demás, volviéndose tema de una nueva expansión. Se trata de la tematización, por la cual, en (1) la falla ortográfica, en (2) la deficiencia del registro escrito, y en (3) la inconsistencia disciplinaria, se volverán a su vez puntos de partida de nuevas aspectualizaciones. Aquí, el efecto de la tematización, lejos de volver más lúcida la mirada al concentrarse en un aspecto, opera una sustitución del todo por la parte, de manera tal que la magnificación de la parte cumple una función de encubrimiento, enmascaramiento de otros aspectos del objeto.

Quisiéramos ahora detenernos en otra operación presente en los fragmentos (2) y (3) y que se superpone a las otras operaciones ya mencionadas: nos referimos a la reformulación. Esta operación da cuenta no sólo de la actividad de corrección de su propio discurso por parte del enunciador sino también del control que este último ejerce sobre la interpretación que el destinatario realizará del enunciado, pues apela a su conocimiento enciclopédico, a un saber presupuesto con el que se busca enlazar una nueva formulación. Además, se ha señalado también, que la reformulación asegura la cohesión semántica (Adam y Revaz, 1989: 84) pues permite asociar unidades por el sentido, lo cual pone en evidencia que se trata de un dispositivo instituido por el propio discurso pues la equivalencia que establece entre las unidades no proviene de la significación meramente léxica sino de los diversos saberes que el discurso pone en circulación. Es esta apertura de la reflexión hacia el carácter discursivo de la reformulación la que permite considerarla como un «cambio de perspectiva enunciativa» (en palabras de Roulet, 1987: 116) pues más que repetir en otros términos un constituyente anterior del discurso apunta a producir una transformación del ángulo focal y, por lo tanto, de la posición que el enunciador reclama de parte del destinatario de su discurso.

Si observamos los fragmentos (2) y (3), advertiremos en ambos la presencia de sucesivas interrogaciones: en (2), «¿Qué es esto? [...] ¿Una adivinanza? [...] ¿Una prueba de ingenio? [...] ¿Una pregunta filosófica? [...] ¿O acaso está preparando el examen de ingreso para la Facultad de 
Medicina?» y en (3) «¿A quién se le ocurre que lo que cuenta en esto es la edad? ¿Qué pensaba? ¿En la maduración afectiva? ¿En el desarrollo psicomotriz?».

La ilación de preguntas, cada una de las cuales contiene una posible aserción, se despliega, en ambos casos, a partir de otra pregunta, en (2) «¿Qué es esto?», y en (3) «¿Qué pensaba?», y las sucesivas preguntas constituyen posibles respuestas ingenuas de un destinatario a su vez ingenuo, a quien el enunciador convoca para exhibir, con la supremacía de quien detenta poder, aquello que desde su perspectiva es una falla, una impericia, en suma, para exhibir la ignorancia, ya sea de normas o de códigos aceptados en el entorno militar, ya de un supuesto saber disciplinario.

La reformulación insistente de las preguntas, que en el caso (2) provienen de un sargento y son dirigidas a un cabo, provocan la respuesta repetitiva del cabo: «No, mi sargento», con lo cual se reafirman las posiciones, esto es, el ironista afianza su autoridad fundada en el conocimiento e imposición de las normas, y el destinatario confirma su sumisión al dar la respuesta tautológicamente implicada por las preguntas. A su vez, en el caso (3), quien pregunta es un experimentado médico militar y su interlocutor un joven médico cuyo resto de pudor o «mala conciencia» todavía alcanza para responder ante las insistentes preguntas, con la frase varias veces reiterada en otros pasajes de la novela: «No discutamos esto delante del conscripto, doctor».

El encadenamiento de preguntas irónicas, cada una de las cuales reformula las anteriores en una suerte de crescendo, muestra la astucia del enunciador para delegar en otro, un enunciador ingenuo, la enunciación de las preguntas y el despliegue de posibles universos de sentido en cuya perspectiva el enunciado podría cobrar alguna significación «poco relevante» en el primer caso, «errónea» en el segundo. Pero los destinatarios de esta estrategia (el cabo, el joven médico) no pueden ponerla en evidencia, son ambos subordinados, y deben aceptar desempeñar el papel de enunciatarios ingenuos que la voz de la autoridad los conmina a adoptar.

Aquí vemos cómo la reformulación discursiva se combina con la figura de la ironía, la cual, a su vez, pone en escena a dos enunciadores, uno, el irónico, que se distancia y no asume lo dicho, y otro, el ingenuo, a quien se atribuye lo enunciado (para decirlo en los términos de Reyes, 1984). La reformulación muestra también la posición dominante del que habla mediante la prolongada extensión de su turno de habla y el tiempo breve dejado al destinatario, además de cancelar toda posibilidad de dar una respuesta que no sea tautológica.

A partir de los fragmentos citados y pensando además en la formación de objetos en general, es posible afirmar también que hay una serie de determinaciones de diverso orden que inciden en su construcción: las que provienen del propio objeto (magnitud, sustancia); las que derivan de sistemas de descripción y categorización ya convencionalizados (reticulación del espacio, del tiempo, y la gran cantidad de clasificaciones y ordenamientos que proporcionan los distintos saberes); las relaciones que el discurso establece con otros objetos (de coexistencia, de exclusión, de yuxtaposición); también, aquellas heredadas de convenciones estilísticas, ya sea que explícita o implícitamente se adopten o se cuestionen; las que se vinculan a la situación de observación (proximidad o distancia — pragmática, 
cognitiva o afectiva - entre sujeto y objeto, desplazamientos, finalidad); y, además, las que provienen de un sistema de valores que puede presentarse con distintos grados de asunción.

En los pasajes de la novela en los que nos hemos detenido sobresalen aquellas determinaciones que emanan de un sistema de valores que se busca exhibir: la transgresión implicada por el modo de aparición del objeto, ya sea bajo un criterio ortográfico, de normas de registro o de un saber disciplinario, y el deber ser del objeto en cuestión perturbado en alguno de esos aspectos, queda puesta de relieve en la irritabilidad que produce en sus diversos observadores. Pero más allá de eso, las pequeñas escenas que tienen como centro la observación de la frase, condensan la presencia simultánea de lo ominoso, la banalidad y un sentido del cumplimiento del deber que se volverá una clave para la lectura de la obra.

\section{La posición del objeto: relación con otros objetos, relación con el sujeto}

A las determinaciones mencionadas debemos sumar el hecho de que hacer aparecer objetos en el discurso mediante el ejercicio de la percepción es una actividad que no sólo pone en relación un observador con lo observado sino que dicha actividad se enmarca en una situación enunciativa, por lo tanto, la imagen de destinatario a quien se ofrece una determinada configuración de objeto también afecta el modo de constituirlo. En este sentido, aquello que hemos denominado como «la frase» y que en tanto tal es blanco de las distintas operaciones ya señaladas, es, más allá de eso, una pregunta: si como unidad de la lengua se deja denominar mediante el término «frase», como objeto propio del discurso no se puede desatender el hecho de que se trata de una pregunta. Ya Bajtín (1982) señalaba, de manera insistente, que todo enunciado $-\mathrm{y}$ no solamente una pregunta- se sustenta en una estructura subyacente de pregunta-respuesta, de aquí el carácter eminentemente dialógico del discurso. Este rasgo se vuelve más notorio en el caso de una pregunta explícita, dado que la pregunta convoca otros discursos, tiende hacia un futuro pues exige la respuesta por venir y se vuelca sobre un pasado porque proviene del campo de discurso que la hace surgir.

$\mathrm{Si}$, en los fragmentos analizados, las operaciones de aspectualización y tematización se ciernen sobre la interioridad de la frase en cuestión, la afectación proyectará la pregunta fuera de sus propios límites para transformarse, de objeto de observación gramatical o disciplinaria, en parte de otro objeto: a la manera del tradicional «motivo» narrativo, la pregunta desencadenará una serie de acontecimientos y será uno de los hilos conductores de la trama. Es así que la pregunta, ahora como parte de la trama, dará inicio al peregrinaje que emprende el protagonista en busca de quien debe dar la esperada respuesta: la pregunta, la minuciosa búsqueda del destinatario, el encuentro, la respuesta, son las fases de un proceso que, junto a otros (la tortura, el fútbol, el servicio militar, por ejemplo) serán puestos bajo la mira del observador.

Todo objeto aparece siempre situado, esto es, en relación con otros objetos y vinculado al ejercicio perceptivo del sujeto. Así, en su relación con otros, el objeto puede aparecer en coexistencia o coordinación (asociación de objetos homogéneos), yuxtaposición (asociación de objetos 
heterogéneos) o subordinación (primero y segundo plano, figura y fondo, etc.), y, en cualquiera de esos casos, asumir significación por sustitución, complementariedad, contradicción, semejanza, etcétera. Y en su relación con el ejercicio de la percepción, el objeto puede transitar de la máxima proximidad a la máxima distancia (espacial, temporal, afectiva, axiológica), puede participar en la generación de diversas modalizaciones en la dimensión cognoscitiva del sujeto (poder/querer/deber ver o saber; no poder/no querer... etc.), puede incidir en las modulaciones de su dimensión pasional (gradaciones de apego/desapego evidenciadas en la prosodia del discurso), puede, en fin, como hemos visto ya, promover distintas aspectualizaciones.

Consideremos, por ejemplo, el posicionamiento del fútbol, en los siguientes pasajes de la novela que nos ocupa:

\section{I}

Para dar pronta respuesta a la inquietud del doctor Padilla, que en el centro de Quilmes la esperaba con urgencia, el sargento Torres me ordenó que ubicara de inmediato al doctor Mesiano. Era necesario que se acercara cuanto antes a la oficina de comunicaciones y se pusiera en contacto con el doctor Padilla.

Pero el doctor Mesiano no aparecía por ninguna parte.

II

La formación de la Argentina: Fillol; Olguín; Galván; Passarella; Tarantini; Ardiles, Gallego, Kempes; Bertoni, Valencia, Ortiz.

III

Recorría los diferentes sectores de la unidad. Primero aquellos donde el doctor Mesiano podía llegar a encontrarse, según las actividades o las preferencias que yo le conocía. En ninguno de esos sitios estaba $[\ldots]$.

$$
\text { IV }
$$

La formación de la Argentina (con especial atención a los nombres de sus integrantes): Fillol, Ubaldo Matildo; Olguín, Jorge Mario; Galván, Luis Adolfo; Passarella, Daniel Alberto [...].

\section{$\mathrm{V}$}

En un primer momento, no quise preguntar a nadie por el doctor Mesiano. De alguna manera, presentía que él había incurrido en una falta, que el hecho mismo que no se lo pudiera ubicar con prontitud ya implicaba una forma de incorrección de su parte [...].

\section{VI}

La formación de la Argentina (con especial atención a las posiciones de sus integrantes): Fillol, arquero; Olguín, marcador de punta por derecha; Galván, marcador central por derecha; Passarella, marcador central por izquierda [...] (Kohan, 2002: 45 y ss.).

La narración de la búsqueda del doctor Mesiano aparece de pronto interceptada por una minuciosa descripción de la composición del equipo de fútbol que llevó Argentina en uno de los partidos del campeonato mundial celebrado en junio de 1978 en ese país. En una primera aproximación, si atendemos al modo de aparición de «la formación de la Argentina», se puede observar que ésta, en tanto objeto de observación, es sometida a un proceso de aspectualización que permite el despliegue de cada una de sus facetas: miembros, nombres, posiciones (en los fragmentos citados), y que se continúan en la novela con la referencia a la procedencia, numeración, fechas de nacimiento, estatura y peso (rasgo que puede ser visto como una suerte de esbozo o anuncio de otro objeto que aparecerá más adelante, la balanza) de sus integrantes. 
Pero lo que más llama la atención del lector, en primer lugar, es el modo de aparición de «la formación de la Argentina», esto es, la interrupción que produce esa minuciosa descripción en el curso de la trama. Esa alternancia insistente entre la narración de la búsqueda y la descripción del equipo de fútbol pone de manifiesto el carácter yuxtapuesto de la vinculación entre ambos dominios u objetos de observación.

Pero esa misma extrañeza que provoca la yuxtaposición de dos objetos heterogéneos no puede sino conducir a realizar alguna asociación que justifique el vínculo que el discurso realiza. Más allá de la anécdota que más adelante enlazará la búsqueda y encuentro del doctor Mesiano con el partido de fútbol que en ese momento estaba teniendo lugar, otra es la relación que sugiere el modo yuxtapuesto de aparición del fútbol en el curso de la narración pues esa yuxtaposición aquí está marcada no sólo por acercar en el discurso dos objetos ajenos uno al otro, sino también por el modo de relación de tales objetos con el sujeto que percibe.

En el caso de «la formación de la Argentina», la exhaustividad en el enlistado de detalles nimios remite al mismo tipo de mirada implicada en el primer parágrafo de la novela (citado aquí en el ejemplo 1), en el cual, decíamos, la detención en un detalle ortográfico colma el radio de alcance de la percepción y exime al observador de captar otros aspectos de lo observado. Se trata de una mirada que se detiene en la superficie de los objetos y los recorre minuciosamente en aquellos aspectos visibles o reconocibles a partir de la evidencia o de un conocimiento fundado en la recolección de datos. Algo semejante sucede más adelante en la novela con la aparición de «la balanza»:

\section{VI}

Más grave que mi demora es su ignorancia, doctor.

La nuestra es una profesión en la que nunca se deja de aprender.

Hablo de ignorancia, doctor, no de perfeccionamiento.

Sería mejor que el conscripto se retire y hablemos a solas usted y yo $[\ldots]$

VII

La balanza tiene un límite máximo de capacidad: son los ciento cincuenta kilos. Por encima de ese límite, no solamente no marca, sino que puede llegar a estropearse su mecanismo.

VIII

Yo estoy perfectamente dispuesto a admitir mis errores, doctor Mesiano. Pero preferiría mantener una conversación privada.

El suyo es, fundamentalmente, un error conceptual. Al pensar en la edad ha pensado en el grado de crecimiento de una persona. Y no es la edad de la persona lo que cuenta, sino su masa corporal, el peso de su cuerpo, para saber si se trata de un cuerpo resistente o no. [...]

\section{IX}

La balanza tiene también un límite mínimo de capacidad: por debajo de los cinco kilos, no pesa. Por supuesto, en esto no hay peligro alguno de roturas o descompensaciones. Se trata meramente de una cuestión de sensibilidad. [...] (Kohan, 2002: 123 y ss.)

Aquí también, el modo yuxtapuesto de aparición de la referencia al instrumento de la balanza, más allá de la vinculación por la trama narrativa, conduce a reflexionar sobre la perspectiva que el 
discurso privilegia, que se asocia con el sujeto propio de un texto descriptivo que reúne datos de interés para fines prácticos y que atienden a la funcionalidad del objeto en cuestión. Otra vez asistimos a una forma de la mirada que se colma con la precisión de los detalles de superficie, de un objeto además marginal, que sólo está ahí para ilustrar, precisamente, una forma de percibir que consiste en multiplicar la minucia de los detalles, saturar el campo perceptivo, y así no poder, impedir o dispensarse de observar otra cosa. Precisamente la ausencia de marcas de subjetividad en estos pasajes unida a la utilización de verbos en presente (tiene, son, hay, se trata...) produce, además del efecto de sentido de validez universal, de verdad incuestionable, el efecto de la figura de un observador atento, conocedor de los mínimos detalles de aquello que pone bajo la mira. La contradicción surge en el momento en que se advierte que estos objetos aspectualizados en exceso y aparentemente agotados en sus posibilidades de significación, están formados como una colección exhaustiva de datos carentes de toda pertinencia para la trama narrativa. Este modo de formación de los objetos mencionados da cuenta, así, de una forma de la mirada, de una manera de ocupar o distraer la atención en minucias que desvían o impiden observar otros aspectos de los fenómenos que darían lugar a otros modos de construcción de los objetos. Se trata de una mirada que pone a distancia los objetos y los recorre en su superficie, los estudia, realiza una suerte de morfología de los mismos, los contempla y los aparta de las relaciones que podrían establecer. Modo de observación que remite a la mirada objetivante que segmenta y aspectualiza de forma hiperbólica para que la exhaustividad de los detalles nimios termine produciendo un vacío de sentido que - en otro nivel de análisis- el narratario está llamado a llenar.

Estas dos determinaciones que aquí hemos apuntado someramente y que requieren de un mayor desarrollo, son fundamentales en la significación de un objeto de percepción en el discurso: la relación que entabla con otros objetos y con el sujeto que percibe. Ambas dan cuenta de la posición del objeto, de su inserción en una red de relaciones que le procuran significación.

\section{Conclusión}

No podríamos dejar de evocar aquí, a propósito de la formación de objetos en esta novela y de su vinculación con el ejercicio perceptivo de un observador, aquella expresión fraguada por Hannah Arendt (1999) sobre «la banalidad del mal». Como se recordará, en el juicio entablado en 1961 contra el criminal de guerra Adolf Eichmann, la autora acentúa el hecho de que lejos de tratarse de una figura monstruosa, perversa o sádica, el acusado manifestó siempre ser una persona normal, ocupada en cumplir estrictamente con su deber consistente en obedecer las órdenes que se le imponían (de donde seguirán, para el caso, ciertas consecuencias sobre la responsabilidad individual en las acciones colectivas). El protagonista de nuestra novela también se considera a sí mismo, de manera constante, como alguien entregado al cumplimiento estricto del deber: la búsqueda incansable del doctor Mesiano por las dependencias militares y por la ciudad es una orden recibida que debe cumplir. En ese peregrinaje se van a rozar apenas escenas vinculadas con la tortura y se destacarán aquellos segmentos en los que se despliega una mirada que se detiene en minucias y en detalles insignificantes. Este 
enmascaramiento y obnubilación que genera la sumisión y entrega de la voluntad propia, individual, a una voluntad ajena, colectiva, y a la que se le atribuye el valor de la razón y del bien más allá de toda explicación posible, conforma el terreno propicio para una actuación individual que se ampara en la ingenuidad, en el no saber ni poder ver, o bien, como decíamos, en la posibilidad de eximirse de considerar el sentido de lo que se pone ante los ojos pues el campo de percepción se colma con colecciones de datos inocuos que saturan la mirada y la comprensión. Si bien Arendt no desarrolla el sentido de la expresión que pone como subtítulo de su libro, consideramos que este enraizamiento del mal en lo cotidiano, en la normalidad (esto es, en lo que es norma y no en la anomia), abre la posibilidad de explicar actuaciones difícilmente comprensibles si se las asimila a fenómenos extraordinarios, monstruosos, fuera de lo común. A pesar de que la frase de Arendt es ambigua y contiene más una provocación a pensar que un sentido claro (pues no se trata de decir que el mal sea banal, como muchos interpretaron) encierra una clara constatación sobre el lugar de origen del mal y de la crueldad, peligrosamente situado fuera de sí (el mal no provendría de una mente malvada) sino en el terreno tan abonado socialmente de la obediencia irreflexiva a la norma y el respeto de la ley en desmedro del ejercicio de la capacidad de juzgar. Por otra parte, entendemos que la manipulación de la percepción está en el centro de las estrategias de captura de la adhesión y del logro de la sumisión, manipulación que se apoya en la saturación del campo de presencia y el consecuente oscurecimiento de las zonas dejadas a distancia del centro de percepción.

La elección de algunos pasajes de la novela de Martín Kohan para mostrar ciertos procedimientos discursivos de la puesta en perspectiva, en particular, la formación de objetos, nos ha permitido poner de manifiesto la importancia de atender a este aspecto del discurso para dar cuenta del proceso de emergencia de la significación, pues el modo de aparición de los objetos en el discurso y la forma de la mirada que convocan son rasgos centrales para reconocer algunos de los múltiples efectos de sentido que produce la lectura de esta novela cuya riqueza significativa queda lejos de ser agotada en estas breves reflexiones centradas sólo en uno de sus aspectos.

\section{Referencias bibliográficas}

Adam J. M., y A. PetitJean (1989): Le texte descriptif. París, Nathan.

ADAM, J. M. y F. REVAZ (1989): «Aspects de la structuration du texte descriptif: les marqueurs d'énumération et de reformulation», Langue française, 81, pp. 59-98.

Apotheloz, D. (1983): «Eléments pour une logique de la description», Degrés, XI, 35-36 (pp. b1b19).

ARENDT, H. (1999): Eichmann en Jerusalén. Un estudio sobre la banalidad del mal. Trad. C. Ribalta. Barcelona, Lumen, $4^{\mathrm{a}}$ ed., 2003.

BAJTín, M. (1982): «Los géneros discursivos», en Estética de la creación verbal. Trad. T. Bubnova. México, Siglo XXI.

Borel, M. J. (1991) «Objets de discours et représentation», Langages, 103. 
228 Tropelías. Revista de Teoría de la Literatura y Literatura Comparada, 25 (2016)

María Isabel Filinich

DASTON, L., ed. (2014): Biografías de los objetos científicos. México, La Cifra.

FABBri, P. (2013): «Semiótica e camuflagem», en A. C. DE OliveIRA, ed., As interações sensíveis.

Ensaios de sociossemiótica a partir da obra de Eric Landowski. São Paulo, CPS / Estaçao das Letras e Cores.

Fontanille, J. (1987): Le savoir partagé Sémiotique et théorie de la connaissance chez Marcel Proust. París/Amsterdam/Philadelphia, Hadès-Benjamins.

_- (1988): «Point de vue: essai de définition discursive», Protée, 16/1-2.

(1994): «El retorno al punto de vista», Morphé, 9-10.

Foucault, M. (1970): La arqueología del saber. Trad. A. Garzón del Camino. México, Siglo XXI.

Hamon, P. (1991): Introducción al análisis de lo descriptivo. Trad. N. Bratosevich. Buenos Aires, EDICIAL.

Kohan, M. (2002): Dos veces junio. Buenos Aires, Debolsillo, $8^{\mathrm{a}}$ ed., 2013.

RABATEL, A. (1998): La construction textuelle du point de vue. Lausanne, Delachaux et Niestlé.

Reyes, G. (1984): Polifonía textual. La citación en el relato literario. Madrid, Gredos. 